\title{
Acanthocéphales de Madagascar
}

\section{(Deuxième Note) \\ Le Genre Pseudoporrorchis Joyeux et Baer 1935}

\author{
Par Yves-J. GOLVAN et Edouard-R. BRYGOO
}

En 1935, Ch. Joyeux et J.-G. Baer décrivaient une nouvelle espèce d'Acanthocéphale parasite de l'intestin d'un Coq de Pagode du Tonkin [Centropus sinensis intermedius (Hume)]. Ils créaient, pour la classer, le genre Pseudoporrorchis Joyeux et Baer 1935 et leur espèce, $P$. houdemeri Joyeux et Baer 1935, était désignée comme espèce-type. Dans leur discussion, ces auteurs proposaient de placer également parmi les Pseudoporrorchis quatre espèces voisines:

- Echinorhynchus bulbocaudatus Southwell et Macfie 1925, parasite de Centropus phasianinus (Latham) au Queensland;

- Echinorhynchus centropusi Tubangui 1933, parasite de Centropus viridis (Scopoli) et de Dasylophus superciliosus (Dumont) aux Philippines ;

- Echinorhynchus rotundatus O. von Linstow 1897, parasite de Centropus toulou (Müller) [=C. madagascariensis (Brisson)] à Madagascar ;

- Echinorhynchus centropi Porta 1910, parasite de Centropus senegalensis (Linné) en Guinée portugaise.

Ainsi se trouvaient groupées cinq espèces d'Acanthocéphales morphologiquement très proches et, qui plus est, toutes parasites de l'intestin de Cuculliformes tropicaux.

Nous avons eu la chance de pouvoir redécrire deux de ces espèces: $P$. centropi (Porta 1910) récolté chez Centropus monachus Rüppell et Centropus sp. au Sénégal (Golvan-1956 a) et $P$. rotundatus (Linstow 1897) récolté chez Centropus toulou à Madagascar (Golvan-1956 b).

La sixième espèce du genre, Pseudoporrorchis teliger Van Cleave 1949, est décrite d'après des exemplaires sexuellement mûrs récoltés dans l'intestin de deux Carnivores de Java: Herpestes javanicus Et. Geoffroy St-Hilaire et Felis minutus javanensis Desmarest.

En 1957, Edmonds assigne Echinorhynchus hylae Johnston 1912 au genre Pseudoporrorchis et décrit Pseudoporrorchis hydromuris Edmonds 1957, parasite de l'in- 
testin d'un Rat d'eau d'Australie; Hydromys chrysogaster (Geoffroy St-Hilaire). Il considère que $P$. centropusi (Tubangui 1933) et $P$. bulbocaudatus (Southwell et Macfie 1925) sont des synonymes de $P$. hylae (Johnston 1912).

En 1957, Das décrit $P$. indicus Das 1957, de l'intestin de Centropus casnopterus (1), Bubo bubo bengalensis (Franklin), Tyto alba javanica (Gmelin) et Milvus migrans govinda aux Indes.

Enfin, en 1958, Petrotschenko considère que l'Echinorhynchus bazae Southwell Macfie 1925, récolté au Queensland chez Baza subcristata (Gould), est un représentant du genre Pseudoporrorchis. Yamaguti (1963) accepte cette suggestion.

Nous pensons qu'avant de décrire une nouvelle espèce malgache que nous venons d'étudier il est utile de reprendre l'essentiel des descriptions des espèces du genre Pseudoporrorchis et de procéder à leur analyse critique.

\section{PSEUDOPORRORCHIS HOUDEMERI JOYEUX ET BAER 1935}

Espèce type du genre Pseudoporrorchis Joyeux et Baer 1935. Viêt-Nam).

Provenance: Intestin (?) de Centropus sinensis intermedius (Hume) du Tonkin (Nord

\section{Description}

Nous devons quelque peu modifier la description originale puisque les auteurs, suivant en cela la nomenclature en usage à l'époque, ont considéré que la «zone inerme », qui s'étend entre la dernière couronne de crochets du rostre et le tronc, fait partie intégrante du proboscis alors que cette portion est actuellement désignée sous le nom de cou et n'est pas comptée dans la longueur du rostre.

« Mâles de $36 \mathrm{~mm}$ de long sur $1 \mathrm{~m}$ de diamètre; femelles de 50 à $55 \mathrm{~mm}$ de long $1,3 \mathrm{~mm}$ de diamètre. Présence, dans la partie antérieure du tronc, d'un renflement séparé de la partie postérieure du corps par une légère constriction annulaire et mesurant $5 \mathrm{~mm}$ de long sur $1,4 \mathrm{~mm}$ de diamètre maximum chez les mâles et $7 \mathrm{~mm}$ de long sur $2 \mathrm{~mm}$ de diamètre maximum chez les femelles Rostre (fig. 1-A) légèrement subterminal, incliné ventralement, en massues, long de 0,60 à $0,66 \mathrm{~mm}$ (au lieu de 0,80 à $1 \mathrm{~mm}$ dans la description originale) sur 0,38 à $0,50 \mathrm{~mm}$ de diamètre, armé de 22 à 24 files longitudinales de onze à douze crochets chacune. D'avant en arrière, la taille des crochets augmente du I au IV, qui est le plus gros, puis diminue assez rapidement $(\mathrm{I}=0,054 \mathrm{~mm} ; \mathrm{II}=0,057 \mathrm{~mm}$; III $=0,085 \mathrm{~mm}$; $\mathrm{IV}=0,092 \mathrm{~mm} ; \mathrm{V}=0,0805 \mathrm{~mm} ; \mathrm{VI}=0,0506 \mathrm{~mm})$. Ces six premiers crochets ont une racine postérieure bien développée (fig. 1-B) portant une légère encoche à son extrémité distale. Les six crochets basaux sont, en fait, des épines de taille plus faible, ne possédant plus que des racines réduites, avec seulement une apophyse supérieure à partir du IX. L'épine XII est simplement implantée sur une petite base. Cou de 0,30 à $0,33 \mathrm{~mm}$ de haut. Réceptacle inséré à la limite du rostre et du cou, possédant une double paroi, long de $1,1 \mathrm{~mm}$. Ganglion

(1) Ce nom d'espèce ne figure pas dans le Catalogue des Cuculliformes de Peters et il doit s'agir d'un synonyme. 
cérébroïde placé à la partie moyenne du réceptacle. Lemnisques nettement plus longs que le réceptacle ( $2 \mathrm{~mm}$ de long) et assez étroits. Testicules très rapprochés l'un de l'autre, ovoïdes, de 1,6 à 1,7 mm de long sur 0,60 mm de diamètre, situés dans le renflement antérieur du tronc lorsque la bourse copulatrice est invaginée. Quatre glandes cémentaires tubuleuses de $22 \mathrm{~mm}$ de long. Organe de Säfftigen (= vésicule séminale dans la description originale), piriforme,
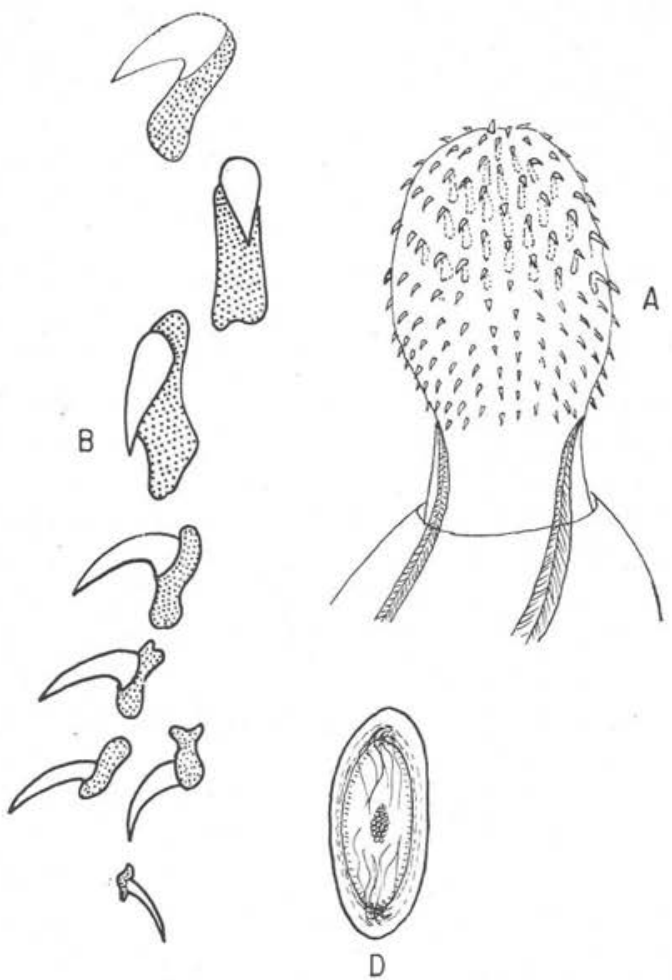

FIG. 1. - Pseudoporrorchis houdemeri Joyeux et Baer 1935 d'après les dessins originaux: A) rostre; B) quelques types de crochets du rostre; C) appareil génital femelle; D) embryophore (il n'y a pas d'échelle dans le travail

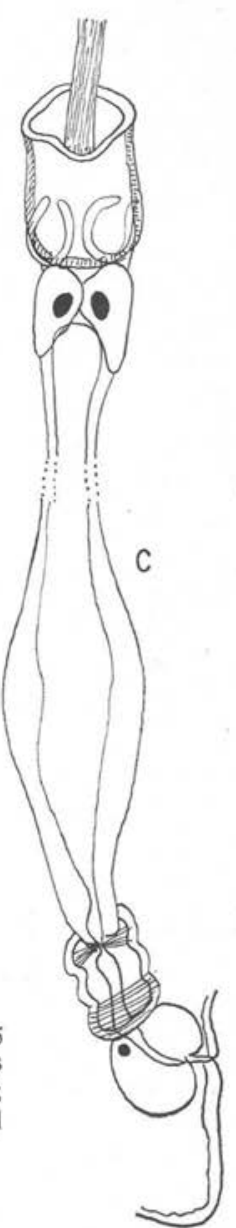

à grosse extrémité supérieure, de $4 \mathrm{~mm}$ de long sur $0,50 \mathrm{~mm}$ de diamètre maximum. Bourse copulatrice renforcée par douze digitations musculaires longitudinales. Appareil éjaculateur entouré d'un puissant sphincter. Deux ventouses copulatrices accessoires d'assez grande taille, disposées symétriquement dans les parois de la bourse. »

Il nous a été impossible de savoir à quelle formation correspondent des « ventouses copulatrices accessoires ». Nous avions pensé qu'il pouvait s'agir des deux diver- 
ticules qui sont souvent annexés à la base de la bourse copulatrice, mais la figure 3 de Joyeux et Baer indique que ce sont des « organes » dépendant du rebord de la bourse. Nous n'avons pas retrouvé d'aspects pouvant rappeler cette disposition chez les mâles de Pseudoporrorchis que nous avons étudié.

« Extrémité postérieure des femelles arrondie, conique, vulve sub-termino-ventrale. Utérus petit, comportant une cloche utérine de $0,40 \mathrm{~mm}$ de long, avec deux diverticules à sa base (fig. 1-C), partie tubulaire de l'utérus de $2 \mathrm{~mm}$ de long, renflée dans son tiers postérieur, où le diamètre atteint $0,32 \mathrm{~mm}$. Vagin de $0,36 \mathrm{~mm}$ de long, à double sphincter. Embryophores (fig. 1-D) de $0,07 \mathrm{~mm}$ de long sur $0,031 \mathrm{~mm}$ de diamètre, à coque externe ornée de saillies vermiculées partant des pôles. Acanthor de $0,049 \mathrm{~mm}$ de long sur $0,021 \mathrm{~mm}$ de diamètre. »

Suivant les opinions formulées par T. Fukui (1929) et S. Yamaguti (1935) à propos du genre Porrorchis Fukui 1929, Joyeux et Baer plaçaient leur nouveau genre dans la sous-famille des Centrorhynchinae A. Meyer 1931, puisqu'en raison de la confusion qui existait alors entre le cou et le rostre proprement dit, ils considéraient que le réceptacle s'insérait à l'intérieur même du proboscis. Nous avons montré (Golvan 1956) qu'il était également peu souhaitable de classer les genres Porrorchis et Pseudoporrorchis dans la sous-famille des Plagiorhynchinae A. Meyer 1931, et proposé la création de la sous-famille des Porrorchinae Golvan 1955. Nous avions, par la suite (Golvan 1960), inséré cette sous-famille dans la famille des Plagiorhynchidae, Golvan 1960, car nous n'avions pas pu avoir connaissance du travail de Petrotschenko (1956) dans lequel avait été proposée la création de la famille des Prosthorhynchidae Petrotschenko 1956 avec laquelle notre nouvelle famille faisait double emploi (Yamaguti 1963).

\section{$* *$ \\ PSEUDOPORRORCHIS CENTROPI (Porta 1910) Joyeux et Baer 1935 (= Echinorhynchus centropi Porta 1910)}

Provenance: Intestin de Centropus senegalensis (Linné) de Guinée portugaise, intestin de Centropus monachus Rüppell et Centropus sp. du Sénégal (Golvan 1956).

\section{Description}

Le texte original de Porta (1910) est le suivant:

«Proboscide quasi sferica; lunga mm 0,6; armata di 20 serie longitudinali di uncini, di questi gli anteriori sono forti et molto arcuati, i posteriori piuttosto deboli e leggermente arcati. Collo inerme, lungo appena $\mathrm{mm} 0,2$. Corpo cilindrico, inerme, terminante in punta nella femina. Uova con triplice invoglio, lunghe $\mathrm{mm} 0,8$ e larghe $\mathrm{mm} 0,3(\times 135)$. Lungh $30,5-40,5 \mathrm{~mm}$. 》 
Les points essentiels de notre redescription de l'espèce peuvent se résumer ainsi :

《 Mâles mesurant de 30 à $35 \mathrm{~mm}$ de long sur $1,4 \mathrm{~mm}$ de diamètre dans la partie antérieure dilatée du tronc, et $1 \mathrm{~mm}$ de large dans la partie postérieure; femelles de $50 \mathrm{~mm}$ de long sur $2 \mathrm{~mm}$ de large en avant et $1,5 \mathrm{~mm}$ de large en arrière. Rostre (fig. 2) pratiquement terminal, avec légère inflexion ventrale, presque parfaitement sphérique, de $1,2 \mathrm{~mm}$ de long sur $1 \mathrm{~mm}$ de large, armé de 20 à 24 files longitudinales de dix à douze crochets chacune, les six à sept crochets antérieurs ont des racines bien développées, les quatre à cinq épines postérieures ont des racines rudimentaires. D'avant en arrière, la taille des crochets et de leurs racines augmente du I au IV qui est le plus gros puis diminue assez rapidement (I $=0,030 \mathrm{~mm} ; \mathrm{II}=0,042 \mathrm{~mm} ; \mathrm{III}=0,053 \mathrm{~mm} ; \mathrm{IV}=0,070 \mathrm{~mm} ; \mathrm{V}=0,060 \mathrm{~mm}$; $\mathrm{VI}=0,048 \mathrm{~mm}$ ). Les racines présentent une sorte de bulbe terminal et une seconde dilatation

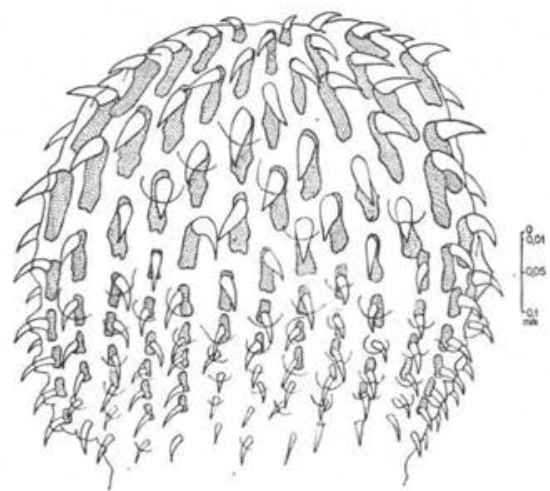

FIG. 2. - Pseudoporrorchis centropi

(Porta 1910) Joyeux et Baer 1935: rostre

immédiatement au-dessus de celui-ci. Epines beaucoup moins arquées que les crochets, poszédant une grosse apophyse supérieure qui persiste seule au niveau de l'épine XII. Cou de $0,25 \mathrm{~mm}$ de haut sur $0,70 \mathrm{~mm}$ de diamètre. Tronc inerme, très allongé, cylindrique, dilaté dans sa partie antérieure, aminci dans sa partie moyenne, dilaté à nouveau dans sa partie toute postérieure, surtout chez les femelles de grande taille. Canaux principaux du système lacunaire reliés par un réseau d'anastomoses à mailles grossièrement polyédriques. Muscles circulaires puissants donnant au tronc une apparence d'annulations pseudo-métamériques. Réceptacle inséré à la limite du rostre et du cou, possédant une double paroi, long de $3,4 \mathrm{~mm}$ sur $0,7 \mathrm{~mm}$ de diamètre. Ganglion cérébroïde placé à la partie moyenne du réceptacle. Lemnisques nettement plus longs que le réceptacle ( $8 \mathrm{~mm}$ de long sur $0,8 \mathrm{~mm}$ de large), en massue à grosse extrémité inférieure. Testicules ovoïdes, placés l'un derrière l'autre, mais non en contact dans le renflement antérieur du tronc lorsque la bourse copulatrice est invaginée, longs de $0,8 \mathrm{~mm}$ sur $0,4 \mathrm{~mm}$ de diamètre. Quatre glandes cémentaires tubuleuses, très longues. Organe de Säfftigen (= vésicule séminale » dans notre texte primitif) très allongé, de $1,5 \mathrm{~mm}$ de long. Bourse copulatrice en position ventrale lorsqu'elle est évaginée. Utérus de $2 \mathrm{~mm}$ de long avec cloche utérine de $0,5 \mathrm{~mm}$ de long suivie d'une portion tubulaire étroite. Vagin court à double sphincter. Vulve ventro-subterminale, s'ouvrant à la base do l'appen- 
dice sus-vulvaire digitiforme terminal. Embryophores de $0,06 \mathrm{~mm}$ de long sur $0,025 \mathrm{~mm}$ de diamètre, possédant une coque externe épaisse ornée de saillies vermiculées partant des pôles. »

\section{PSEUDOPORRORCHIS ROTUNDATUS (O. v. Linstow 1897) Joyeux et Baer 1935}

(=Echinorhynchus rotundatus Otto von Linstow 1897)

Provenance : intestin de Centropus toulou (Müller) (= Centropus madagascariensis (Brisson) de Madagascar.

\section{Description}

Le texte original de Linstow (1897) est ainsi traduit par de Marval (1905) :

« Corps ridé, mesurant $38 \mathrm{~mm}$ de long sur 0,95 mm de large. Rostre armé de dix cercles transverses de quinze crochets chacun. Les premiers mesurant $0,084 \mathrm{~mm}$, les derniers ne mesurant plus que $0,035 \mathrm{~mm}$ et n'ayant pas de racines. Chez les mâles, le corps se termine en boule. Les œufs ont deux coques et mesurent $0,06 \mathrm{~mm}$ de long sur $0,031 \mathrm{~mm}$ de large. 》

Nous avons redécrit l'espèce (Golvan 1956-b) et en avons depuis reçu de nombreux spécimens provenant de $C$. caerulea $\left(\mathrm{n}^{\circ}\right.$ 223/62) tué à Périnet le 3 juillet 1962, Coua cristata pyropyga $\left(\mathrm{n}^{\circ}\right.$ 200/60) tué à Andresoro, Tananarive, en février 1961, C. toulou $\left(n^{\circ}\right.$ 230/62) tué à Périnet le 4 juillet 1962, Coua caerulea (Linné) ( $\left.\mathrm{n}^{\circ} 123 / 62\right)$ tué à Périnet le 8 avril 1962, C. caerulea $\left(\mathrm{n}^{\circ}\right.$ 223/62) tué à Périnet le 3 juillet 1962, Coua cristata pyrogyga $\left(\mathrm{n}^{\circ} 327 / 62\right)$ tué à Evary le 30 septembre 1962 , Coua reynaudi Pucheran $\left(\mathrm{n}^{\circ} 420 / 62\right)$ tué à Périnet le 9 décembre 1962), $C$. caerulea (Linné) ( $\left.{ }^{\circ} 11 / 63\right)$ tué à Sahafonjana (leg. de l'I.R.S.M.). Nous l'avons nous-même, en collaboration avec Alain-G. Chabaud, récolté chez C. toulou et C. reynaudi à Périnet le 22 avril 1964.

Les juvéniles de cette espèce ont été récoltés dans le péritoine de Mantella betsileo $\left(n^{\circ} 15 / 61\right)$ provenant de la Côte Est de Madagascar, et dans l'épaisseur de la paroi intestinale de Mimophis mahafalensis $\left(\mathrm{n}^{\circ}\right.$ 186/62) capturé à Fianarantsoa le 15 mars 1962. Le premier hôte intermédiaire est très certainement un insecte. Nous avons tenté de réaliser expérimentalement le cycle en faisant ingérer à des Criquets migrateurs et à des Blattes des embryophores provenant de la cavité générale des femelles récoltées chez $C$. toulou à Périnet. Cette expérimentation est actuellement en cours. Les Batraciens doivent pouvoir servir d'hôtes de réenkystement efficaces puisqu'à l'autopsie d'un Centropus nous avons trouvé des débris de Microhylidae encore identifiables dans l'estomac.

Pour l'essentiel, notre redescription de l'espèce peut se résumer de la façon suivante :

«Mâles mesurant de 30 à $35 \mathrm{~mm}$ de long sur $1,5 \mathrm{~mm}$ de large dans la partie antérieure dilatée du tronc et $1 \mathrm{~mm}$ de diamètre dans la partie moyenne. Femelles de 32 à $48 \mathrm{~mm}$ de long sur $2 \mathrm{~mm}$ de large en avant et $1,5 \mathrm{~mm}$ de large en arrière. Rostre (fig. 3-B) terminal, 
FIG. 3. - Pseudoporrorchis rotundatus (O.v. Linstow 1897) Joyeux et Baer 1935 de Coua reynaudi : A) individu mâle sexuellement mûr ; B) rostre ; C) types de crochets du rostre

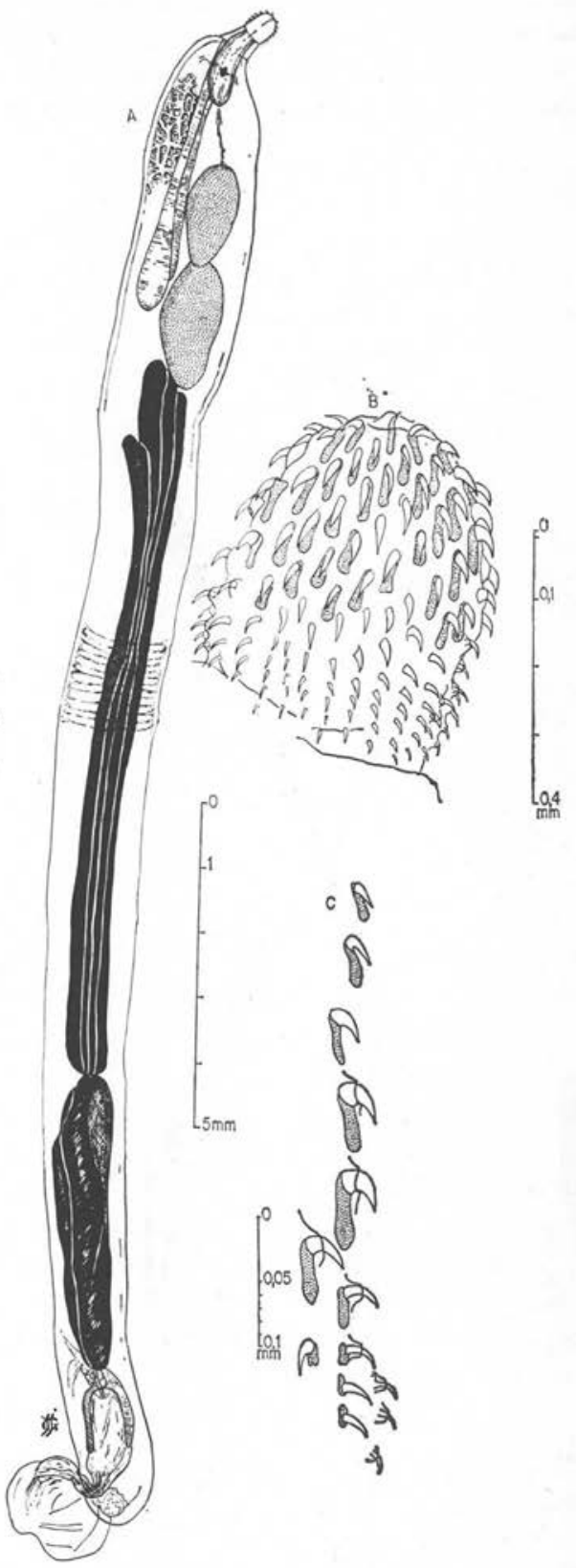


presque parfaitement sphérique, de 0,65 à $0,75 \mathrm{~mm}$ de long sur 0,55 à $0,60 \mathrm{~mm}$ de diamètre, armé de 28 à 32 files longitudinales de dix à onze crochets chacune, les cinq crochets antérieurs ont des racines bien développées (fig. 3-C), les cinq à six épines postérieures ont une racine réduite présentant une apophyse supérieure, sauf celles de la dernière couronne qui
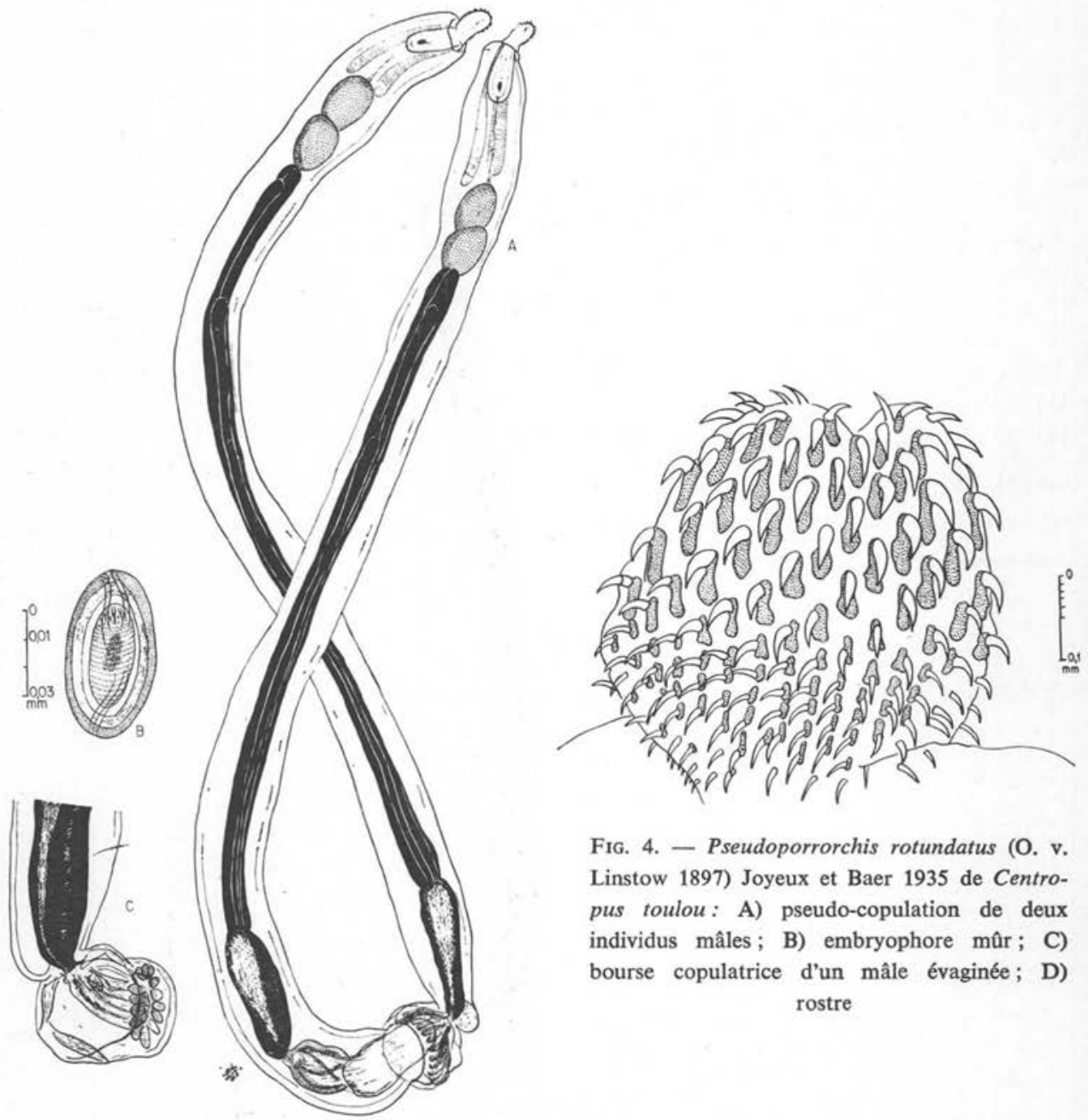

FIG. 4. - Pseudoporrorchis rotundatus (O. v. Linstow 1897) Joyeux et Baer 1935 de Centropus toulou: A) pseudo-copulation de deux individus mâles; B) embryophore mûr; C) bourse copulatrice d'un mâle évaginée; D) rostre

s'insèrent sur une petite base d'aspect granuleux. D'avant en arrière la taille des crochets et de leurs racines augmente du I au III qui est le plus gros, puis diminue rapidement (I = $0,025 \mathrm{~mm} ; \mathrm{II}=0,040 \mathrm{~mm} ; \mathrm{III}=0,065$ à $0,070 \mathrm{~mm} ; \mathrm{IV}=0,045$ à $0,050 \mathrm{~mm} ; \mathrm{V}=$ $0,040 \mathrm{~mm}$ ). Les racines présentent un bulbe terminal non surmonté d'une seconde dilatation comme chez $P$. centropi. Epines beaucoup moins arquées que les crochets, possédant une 
grosse apophyse supérieure au niveau de l'épine VII ou VIII. Cou de 0,25 à 0,275 mm de haut sur $0,20 \mathrm{~mm}$ de diamètre. Tronc inerme, très allongé, cylindrique, dilaté dans sa partie antérieure, aminci dans sa partie moyenne, dilaté à nouveau, dans sa partie toute postérieure, surtout chez les femelles gravides de grande taille. Canaux principaux du système lacunaire latéraux, reliés par un réseau d'anastomoses à mailles grossièrement polyédriques. Assise musculaire circulaire pariétale formée d'anneaux puissants donnant au tronc l'apparence d'annulations à disposition pseudo-métamérique. Réceptacle inséré à la limite du rostre et du cou, possédant une double paroi, long de 1,3 à $1,6 \mathrm{~mm}$ sur 0,5 à $0,7 \mathrm{~mm}$ de diamètre. Ganglion cérébroïde placé à la partie moyenne du réceptacle. Lemnisques nettement plus longs que le réceptacle ( 3 à $5 \mathrm{~mm}$ de long), en massue, à grosse extrémité inférieure. Testicules ovoïdes, placés l'un derrière l'autre mais non en contact (fig. 4-A) dans le renflement antérieur du tronc lorsque la bourse copulatrice est invaginée, longs de $1 \mathrm{~mm}$ sur 0,5 à $0,7 \mathrm{~mm}$ de diamètre. Quatre glandes cémentaires tubuleuses, très longues. Souvent une paire de glandes est un peu plus courte que l'autre paire. Vésicule séminale ou ampoule déférentielle de $1,8 \mathrm{~mm}$ de long. Organe de Säfftigen de $1,5 \mathrm{~mm}$ de long. Bourse copulatrice en position ventrale lorsqu'elle est évaginée, possédant une quinzaine de digitations musculaires dans l'épaisseur de sa paroi (fig. 4-C). Appareil génital femelle mesurant $1,9 \mathrm{~mm}$ de long, comprenant une cloche utérine de $0,3 \mathrm{~mm}$ de long sur $0,23 \mathrm{~mm}$ de large, centrée par le ligament antéro-postérieur, une portion tubulaire de $1,4 \mathrm{~mm}$ de long dont la portion terminale contient l'entonnoir vaginal de $0,375 \mathrm{~mm}$ de long, un vagin comportant deux forts sphincters (fig. 5). Vulve ventro-subterminale, s'ouvrant à la base de l'appendice sus-vulvaire digitiforme terminal. Embryophores (fig. 4-B) de 0,06 à $0,065 \mathrm{~mm}$ de long sur 0,03 à $0,003 \mathrm{~mm}$ de diamètre, à coque externe épaisse, ornée de saillies vermiculées partant des deux pôles. Acanthor à cuticule épineuse (fig. 6). »

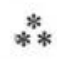

\section{PSEUDOPORRORCHIS TELIGER Van Cleave 1949}

Provenance : Intestin de Herpestes javanicus Et. Geoffroy Saint-Hilaire et de Felis minutus javaensis Desmarest à Batavia (Indonésie).

\section{Description}

Voici l'essentiel de la description de Van Cleave :

« Corps long, cylindrique, de $48 \mathrm{~mm}$ de long chez les femelles et de $36 \mathrm{~mm}$ chez les mâles. Diamètre maximum chez les individus sexuellement mûrs compris entre 1,6 et $3,1 \mathrm{~mm}$. Proboscis court, claviforme, avec une partie distale ovoïde et une partie basale un peu plus large (fig. 7), mesurant approximativement 0,75 à $0,86 \mathrm{~mm}$ de long sur 0,48 à $0,60 \mathrm{~mm}$ de diamètre maximum, armé de 24 à 28 files longitudinales de neuf à dix crochets chacune. »

Le texte de Van Cleave concernant la description de la morphologie des crochets et de leurs racines est assez obscur et il nous paraît qu'il doive être interprété comme suit :

« Chaque file comporte trois crochets vrais à racines postérieures et, alternativement, six ou sept épines dont les racines sont réduites à une apophyse antérieure. Les crochets vrais ont 
une lame falciforme de 0,084 à $0,095 \mathrm{~mm}$ de long pour un diamètre d'au moins $0,030 \mathrm{~mm}$ au point d'insertion de la lame sur la racine. Les racines sont extrêmement longues (jusqu'à $0,105 \mathrm{~mm}$ ) celles des crochets II et III- $a$ portent une paire d'apophyses latérales aliformes. Les racines des crochets III- $b$, beaucoup plus petits, ont une racine bien plus courte et dont l'extrémité postérieure est élargie, rappelant la forme d'un fer de hache. Les épines IV possèdent une très courte racine postérieure mais surtout une longue apophyse supérieure se terminant en haut par une bifurcation formant deux «cornes » latérales. Les épines de la cou-
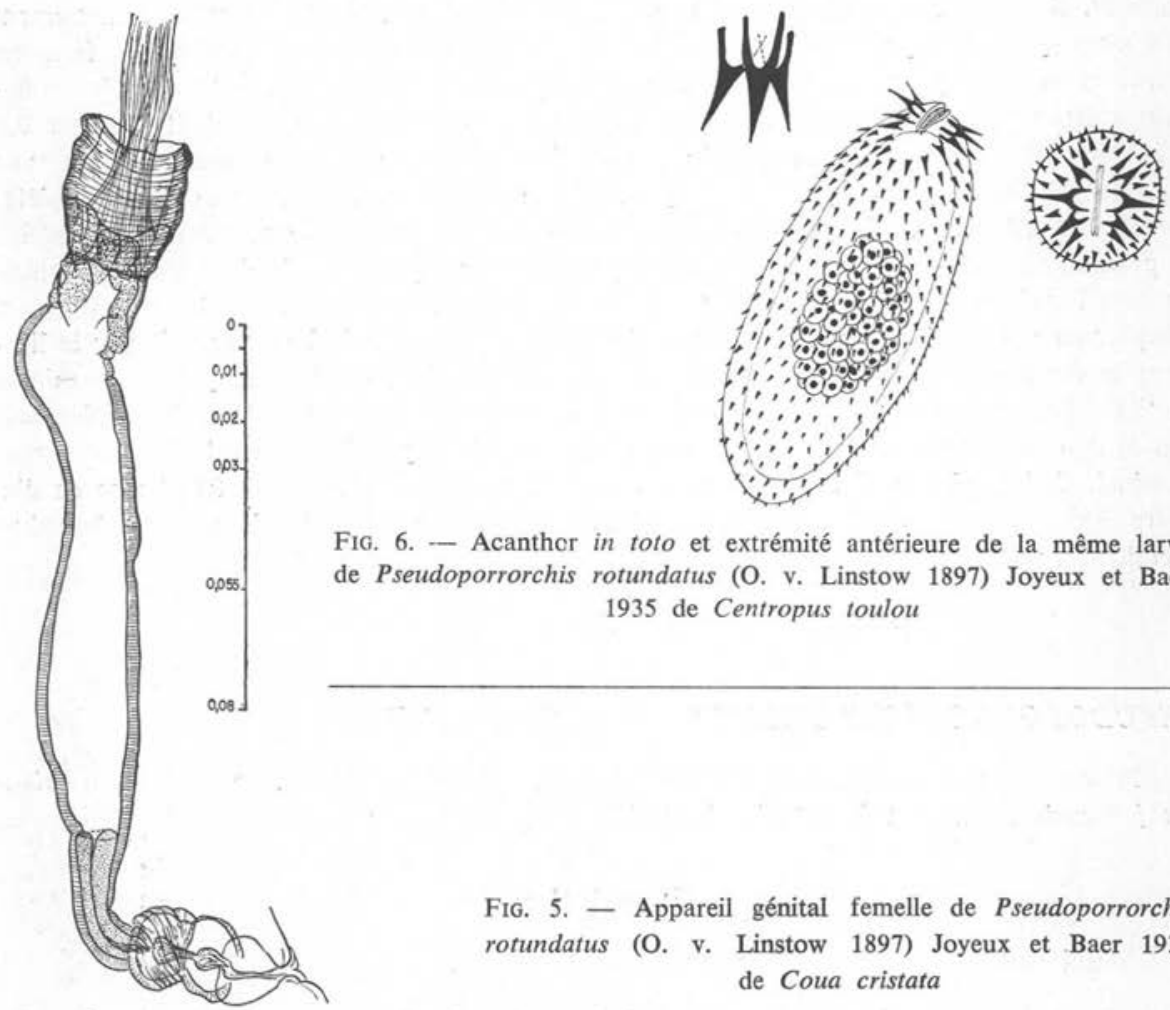

Fic. 6. - Acanther in toto et extrémité antérieure de la même larve de Pseudoporrorchis rotundatus (O. v. Linstow 1897) Joyeux et Baer 1935 de Centropus toulou

ronne basale possèdent une racine rudimentaire discoïde sur laquelle s'insère l'épine. Cou tronc-conique d'environ $0,34 \mathrm{~mm}$ de long. Réceptacle en sac clos, cylindrique, à double paroi. Ganglion cérébroïde placé très en avant dans le réceptacle, immédiatement en dessous de la limite supérieure du tronc. Rétinacles passant latéralement du ganglion cérébroïde dans la paroi musculaire du tronc. Lemnisques un peu plus longs que le réceptacle, souvent difficiles à distinguer. Testicules allongés, ellipsoïdaux, généralement placés loin l'un de l'autre. Glandes cémentaires formant une masse compacte dans laquelle il n'est pas possible d'individualiser chaque glande, d'en discerner la forme et même de connaître leur nombre. Embryophores mûrs de 0,053 à $0,059 \mathrm{~mm}$ de long sur 0,020 à $0,027 \mathrm{~mm}$ de diamètre. » 

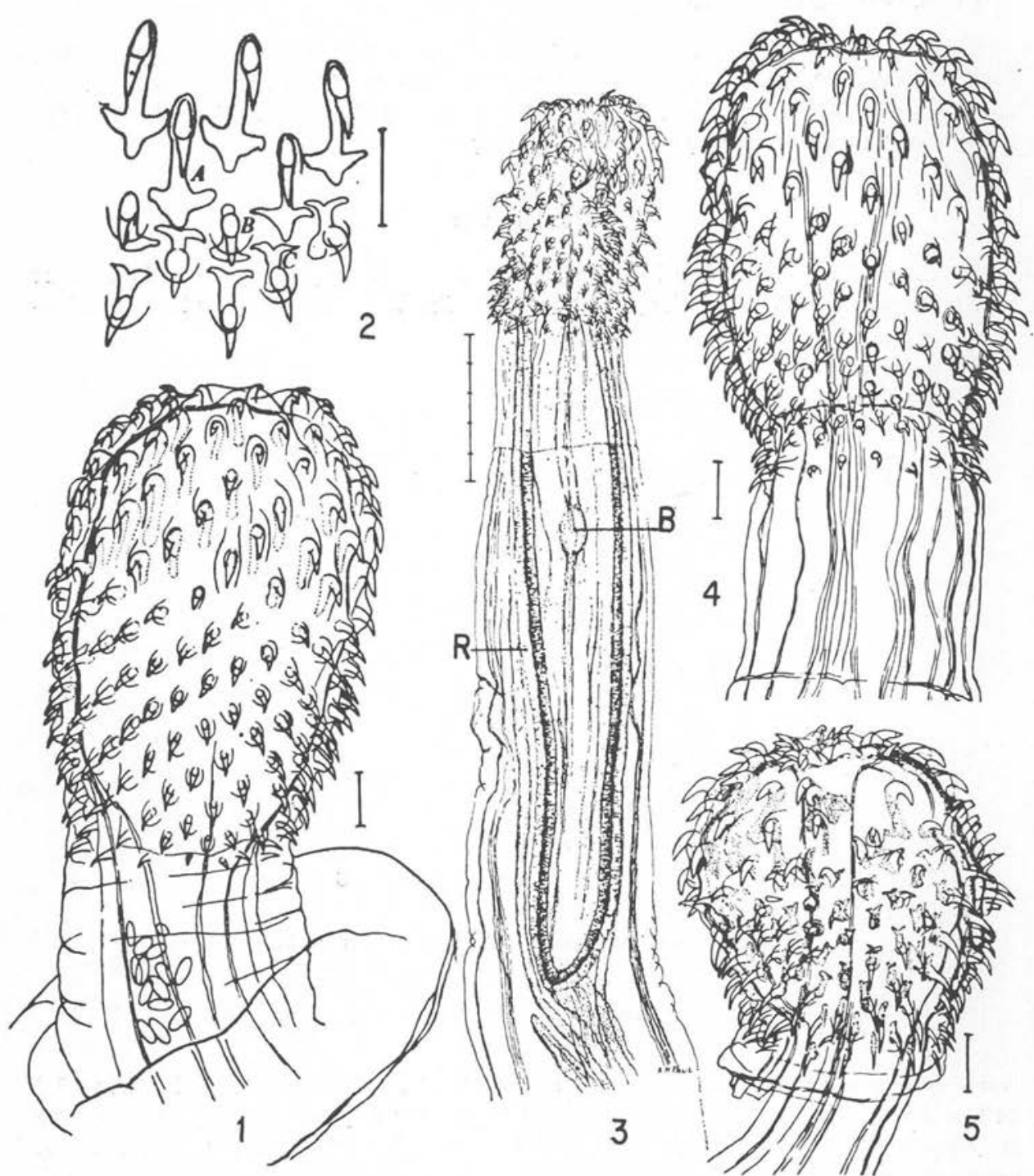

FIG. 7. - Pseudoporrorchis teliger Van Cleave 1949, reproduction des dessins originaux : 1) praesoma d'holotype femelle provenant de Herpestes; 2) forme et disposition des crochets sur la partie latérale du rostre ; 3) praesoma et partie antérieure du tronc d'un spécimen immature provenant de Fetis minutus javanicus; 4) proboscis et cou d'un individu mûr provenant de Herpestes;

5) proboscis partiellement invaginé d'un individu immature provenant de Herpestes 


\section{PSEUDOPORRORCHIS INDICUS DAS 1957}

Provenance: Les adultes ont été trouvés dans l'intestin de Centropus castanopterus, Bubo bubo bengalensis (Franklin), Tyto alba javanica (Gmelin) et Milvus migrans govinda. Les juvéniles proviennent du péritoine d'un Lycodon sp. Les spécimens ont été trouvés en Inde, à Amravati (Etat de Bombay) pour ceux du Centropus.

\section{Description}

\&Les spécimens mûrs sont de grande taille, $46 \mathrm{~mm}$ de long sur $1,4 \mathrm{~mm}$ de diamètre pour les femelles, et $28 \mathrm{~mm}$ de long sur $1,2 \mathrm{~mm}$ de diamètre maximum pour les mâles. Pro-

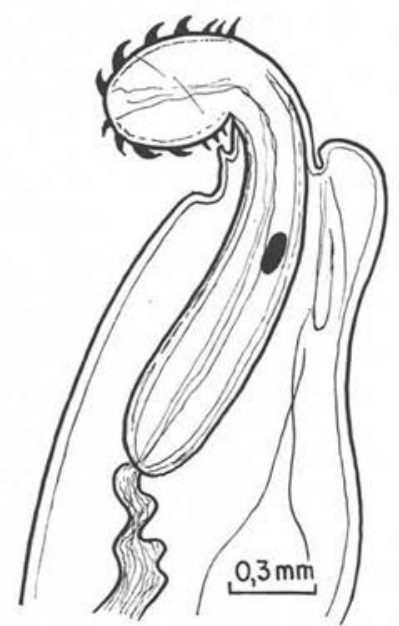

A
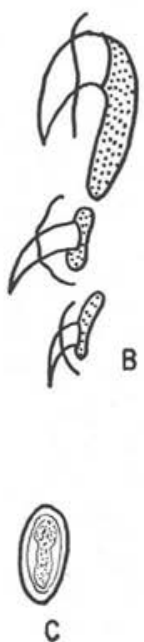

C

FIG. 8. - Pseudoporrorchis indicus Das 1957. Figures faites d'après les clichés photographiques qui illustrent l'article original: A) praesoma ; B) types de crochets du rostre; C) embryophore

boscis_ovoïde de $0,4 \mathrm{~mm}$ de long sur $0,3 \mathrm{~mm}$ de diamètre maximum, armé d'environ 20 à 24 files longitudinales de huit à neuf crochets (fig. 8). Il y a quatre (ou cinq) crochets vrais it quatre (ou cinq) épines. Les crochets antérieurs possèdent une lame dont la partie libre mesure de 0,04 à $0,06 \mathrm{~mm}$ de long pour un diamètre de 0,02 à 0,03 au point d'insertion de la lame sur la racine. Cette racine postérieure a une forme simple («shoe-shaped» dans le texte), et est bien plus longue que la lame, mesurant de 0,07 à $0,09 \mathrm{~mm}$ de long. La racine de l'épine $\mathrm{V}$ est de taille réduite, la lame s'insère au milieu de la hauteur de cette racine, si bien qu'il existe une apophyse postérieure et une supérieure. Les trois ou quatre dernières épines ont une racine bien plus petite et leurs lames mesurent entre 0,025 et $0,030 \mathrm{~mm}$ de long. Les racines n'ont qu'une apophyse antérieure. Cou de $0,4 \mathrm{~mm}$ de haut sur $0,18 \mathrm{~mm}$ de 
diamètre. Réceptacle cylindrique de $1,35 \mathrm{~mm}$ de long pour un diamètre maximum de $0,30 \mathrm{~mm}$, possédant une double paroi de $0,05 \mathrm{~mm}$ d'épaisseur. Ganglion cérébroïde placé dans la moitié antérieure du réceptacle $(0,15 \mathrm{~mm}$ de long sur $0,06 \mathrm{~mm}$ de diamètre), formé d'une grappe de cellules nerveuses. Lemnisques longs et tubuleux de $2,55 \mathrm{~mm}$ de long, dont l'extrémité postérieure atteint presque les testicules. Testicules ovoïdes, placés très près l'un de l'autre, sensiblement égaux (1,20 mm de long sur $0,54 \mathrm{~mm}$ de diamètre). Quatre glandes cémentaires longues et tubuleuses, de $0,35 \mathrm{~mm}$ de diamètre maximum, auxquelles fait suite un réservoir commun placé dans la partie postérieure du tronc et en connexion avec la bourse copulatrice. Embryophores ovoïdes ayant de 0,032 à $0,050 \mathrm{~mm}$ de long, sur 0,011 à $0,020 \mathrm{~mm}$ de diamètre. (Les dimensions les plus petites correspondent manifestement à des œufs immatures).

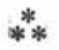

\title{
PSEUDOPORRORCHIS (?) BAZAE (Southwell et Macfie 1925)
}

\section{Petrotschenko 1958}

\author{
(= Echinorhynchus bazae Southwell et Macfie 1925)
}

Provenance : Intestin de Baza subcristata (Gould) de Townsville (Queensland).

\section{Description}

La traduction littérale du texte original est la suivante :

«Le mâle mesure $33 \mathrm{~mm}$ de long et son diamètre maximum est de $2 \mathrm{~mm}$. Les deux femelles sont incomplètes, les fragments mesurent respectivement 45 et $50 \mathrm{~mm}$ de long et environ $2 \mathrm{~mm}$ de large. Corps rugueux, sans épines. Proboscis court et large, un peu rétréci près de sa partie moyenne, plus large dans sa moitié basale, avec une extrémité antérieure arrondie. Chez le mâle il mesure $0,9 \mathrm{~mm}$ de long sur $0,64 \mathrm{~mm}$ de diamètre, et chez les femelles $1,2 \mathrm{~mm}$ de long sur $0,7 \mathrm{~mm}$ de large. Les crochets, qui s'étendent jusqu'à la base du rostre, sont disposés radialement sur environ 38 à 41 files antéro-postérieures composées chacune de douze ou treize crochets. Les crochets des deux tiers antérieurs sont plus grands que ceux du reste du rostre et ont de longues racines rectangulaires. Les plus grands d'entre eux (les antérieurs) mesurent environ $0,090 \mathrm{~mm}$ de long. Le réceptacle est inséré à la base du proboscis. Il n'y a pas de cou. Chez le mâle le réceptacle mesure $1,4 \mathrm{~mm}$ de long sur $0,76 \mathrm{~mm}$ de diamètre, et chez les femelles $1,78 \mathrm{~mm}$ de long sur $0,70 \mathrm{~mm}$ de diamètre. Le ganglion cérébroïde se trouve dans le tiers moyen du réceptacle. Les lemniques sont un peu plus de deux fois plus longs que le réceptacle. Les testicules sont placés juste en arrière du réceptacle et sont disposés obliquement l'un derrière l'autre. Ils mesurent environ $1,5 \mathrm{~mm}$ de long sur $0,66 \mathrm{~mm}$ de diamètre. Il y a apparemment six glandes cémentaires très longues, cylindriques, commençant immédiatement en arrière du testicule postérieur. Les embryophores mesurent $0,078 \mathrm{~mm}$ de long sur $0,041 \mathrm{~mm}$ de diamètre. Ils ne présentent pas de hernies polaires. 》

Cette description assez rudimentaire ne s'accompagne d'aucune figure et il est impossible de savoir avec quelques chances d'exactitude à quel genre d'Acanthocéphales parasites de Rapaces peuvent appartenir les exemplaires étudiés par Southwell et Macfie. 
Petrotschenko (1958) en a fait des Pseudoporrorchis, avec quelque vraisemblance, semble-t-il ; mais, en fait, ceci ne pourra être affirmé qu'après examen des types s'ils existent encore ou par l'étude d'un matériel nouveau provenant du même hôte capturé dans la même région.

\section{$* *$}

\section{PSEUDOPORRORCHIS HYDROMURIS Edmonds 1957}

Provenance: Intestin grêle d'Hydromys chrysogaster Et. Geoffroy Saint-Hilaire capturé à Innisfail (Queensland).

\section{Description}

《Mâles de 14 à $17 \mathrm{~mm}$ de long, femelles de 12 à $19 \mathrm{~mm}$. Tronc cylindrique, tendant à s'amincir quelque peu vers les deux extrémités. Diamètre maximum situé dans le tiers antérieur du tronc et atteignant 1,1 à $1,5 \mathrm{~mm}$ chez les mâles et 1,5 à $2,2 \mathrm{~mm}$ chez les femelles. Proboscis relativement petit et pratiquement sphérique, de 0,40 à $0,46 \mathrm{~mm}$ de diamètre, armé d'environ 26 files longitudinales de sept ou huit crochets chacune. Le crochet II ou III de chaque file est le plus gros et possède une racine à direction postérieure bien développée (longueur de la lame $=0,04$ à $0,05 \mathrm{~mm}$; longueur de la racine $=0,06$ à $0,07 \mathrm{~mm}$ ). Les épines V, VI, VII, VIII voient se réduire progressivement le prolongement postérieur de leurs racines tandis que l'apophyse antérieure apparaît et augmente progressivement de longueur. Les extrémités postérieures des racines sont quelque peu dilatées en bulbe (fig. 9). Cou court d'environ $0,2 \mathrm{~mm}$ de long. Réceptacle de $1,4 \mathrm{~mm}$ de long sur $0,35 \mathrm{~mm}$ de diamètre, à double paroi, inséré immédiatement en-dessous de la dernière couronne d'épines. Deux testicules ellipsoïdaux, de 1,1 à $1,8 \mathrm{~mm}$ de long sur 0,6 à $0,8 \mathrm{~mm}$ de diamètre, placés l'un derrière l'autre dans le tiers antérieur du tronc. Six glandes cémentaires longues et tubuleuses, étroitement appliquées les unes contre les autres. Extrémité postérieure de la femelle arrondie, mais non dilatée, ne portant pas d'appendice sus-vulvaire. Orifice génital femelle terminal. Embryophores mûrs ellipsoïdaux à coque externe épaisse de 0,068 à $0,075 \mathrm{~mm}$ de long sur 0,032 à $0,036 \mathrm{~mm}$ de diamètre. Pas de hernies polaires. Les coupes longitudinales montrent, dans les deux sexes, une pseudosegmentation interne. » (Il s'agit sans doute des anneaux de l'assise musculaire pariétale circulaire).

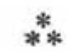

\section{PSEUDOPORRORCHIS CENTROPUSI}

\section{(Tubangui 1933) Joyeux et Baer 1935}

\section{(= Echinorhynchus centropusi Tubangui 1933)}

Provenance : Intestin grêle de Centropus viridis (Scopoli) et de Dasylophus superciliosus (Dumont). Le parasite est fréquent chez le Coucal et rare chez le Coucou car Tubangui ne l'a pas retrouvé lors de l'autopsie de plusieurs Dasylophus. Les localités de récolte sont Novaliches, Rizal et Los Banos, Laguna (île de Luzon) (Philippines). 

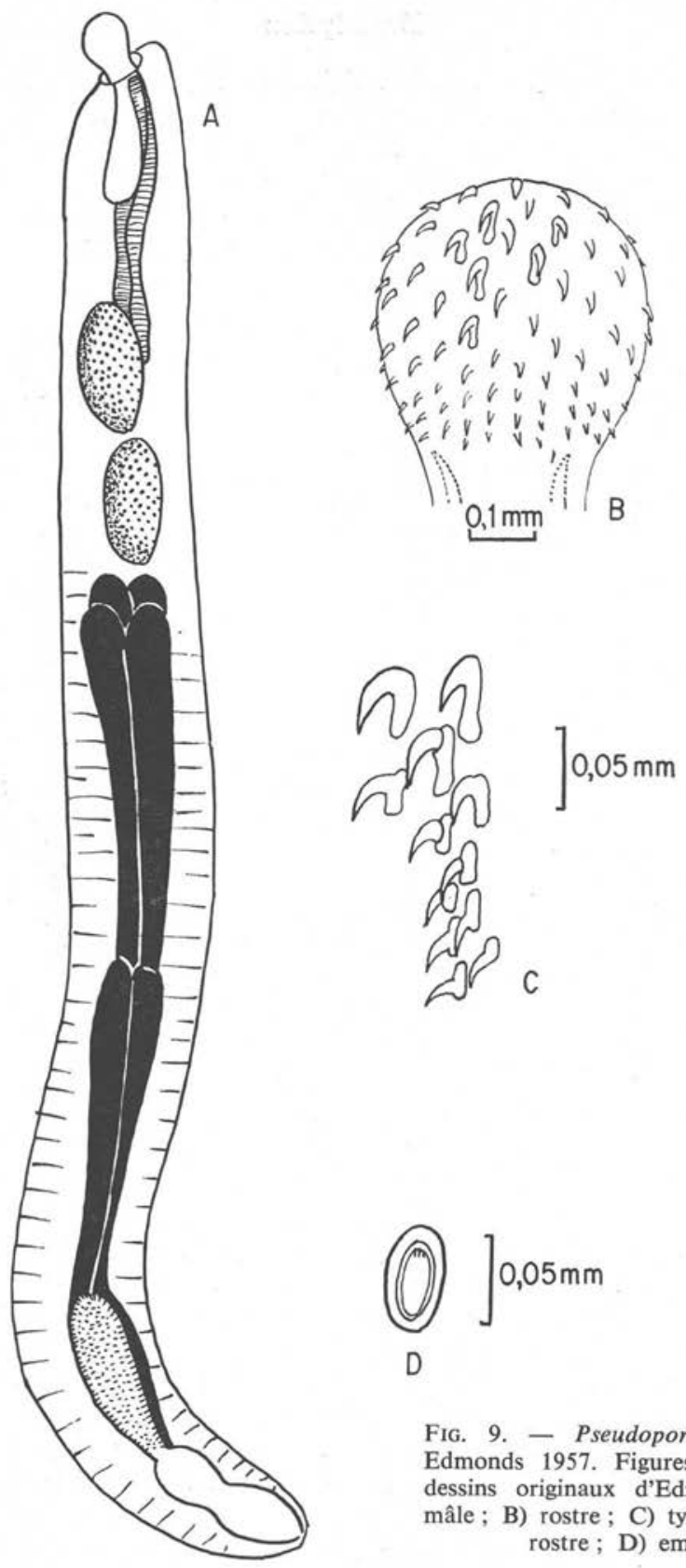

FIG. 9. - Pseudoporrorchis hydromuris Edmonds 1957. Figures faites d'après les dessins originaux d'Edmonds. A) individu mâle ; B) rostre ; C) types de crochets du rostre; D) embryophore 


\section{Deseription}

«Corps allongé, cylindrique, à surface légèrement rugueuse. Le sixième ou le septième antérieur du tronc est dilaté, surtout chez les femelles, est séparée du reste du tronc par une constriction annulaire. Mâles de 11,5 à $14,5 \mathrm{~mm}$ de long sur 0,80 à $1 \mathrm{~mm}$ de diamètre ; femelles de 20 à $30 \mathrm{~mm}$ de long sur 0,90 à $1,25 \mathrm{~mm}$ de diamètre. Proboscis subsphérique à ovoïde, de 0,40 à $0,50 \mathrm{~mm}$ de long sur 0,40 à $0,46 \mathrm{~mm}$ de diamètre, armé de 16 à 18 couronnes de huit à dix crochets chacune [Edmonds (1957), qui a réexaminé
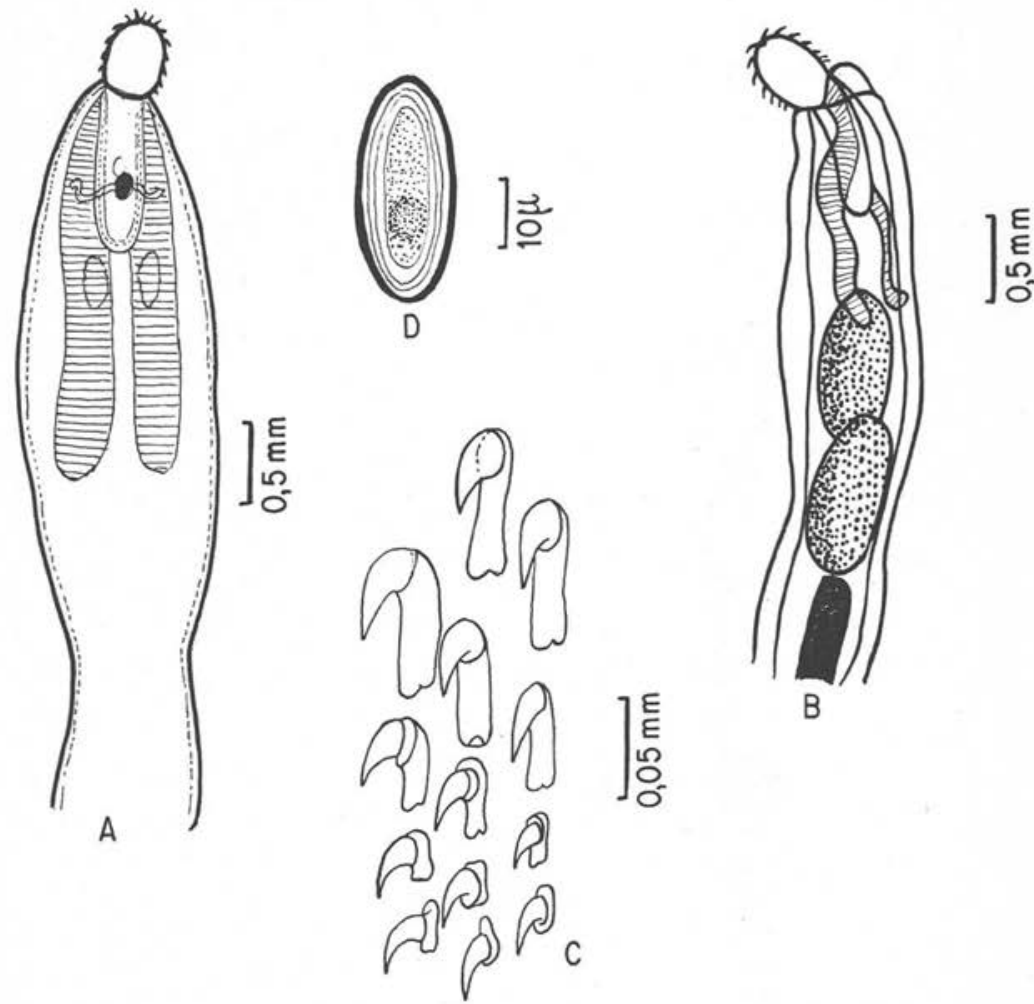

\section{ह}

FIG. 10. - Pseudoporrorchis centropusi (Tubangui 1933) Joyeux et Baer 1935.

Figures faites d'après les dessins originaux de Tubangui : A) praesoma; B) praesoma d'un mâle; C) types de crochets du rostre; D) embryophore

les types de Tubangui, écrit : « 26 files longitudinales de huit à dix crochets chacune ».]. Les zrochets du tiers antérieur du proboscis sont les plus gros et mesurent 0,087 à $0,102 \mathrm{~mm}$ de long (racine comprise), les autres " crochets" (ce sont en fait des " épines ") sont plus petits et mesurent 0,038 à $0,042 \mathrm{~mm}$ de long et ont des racines très réduites. Cou très court, inerme. Réceptacle à double paroi, de 1 à $1,20 \mathrm{~mm}$ de long sur 0,30 à $0,38 \mathrm{~mm}$ de diamètre. Ganglion cérébroöde placé en arrière de la moitié de la longueur du réceptacle. Lemnisques étroits ou moyennement larges, possédant un unique gros tuyau, ayant environ deux fois la 
longueur du réceptacle et atteignant le pôle antérieur du testicule antérieur. Testicules ovoïdes à ellipsoïdaux, l'un derrière l'autre, parfois se superposant plus ou moins, de 0,90 à $1,04 \mathrm{~mm}$ de long sur 0,50 à $0,52 \mathrm{~mm}$ de diamètre, placés dans la partie antérieure du tronc à 0,30 ou $1 \mathrm{~mm}$ du fond du réceptacle et immédiatement en avant de la striction annulaire du tronc. Glandes cémentaires longues mais dont on ne peut connaître le nombre avec exactitude. Organe de Säfftigen (=" cement reservoir" dans le texte) allongé, de 2,40 mm de long sur $0,30 \mathrm{~mm}$ de large.. Bourse copulatrice en cloche de 1 à $1,12 \mathrm{~mm}$ de long sur $0,94 \mathrm{~mm}$ de large. Extrémité postérieure des femelles un peu dilatée se terminant en pointe conique. Cloche utérine relativement petite. Embryophores à trois coques concentriques, mesurant 0,029 à $0,0375 \mathrm{~mm}$ de long sur 0,0145 à $0,0187 \mathrm{~mm}$ de diamètre (il s'agit d'embryophores immatures, les dimensions à maturité données par Edmonds 1957 sont: $0,05 \mathrm{~mm}$ de long sur $0,023 \mathrm{~mm}$ de diamètre). »

\section{PSEUDOPORRORCHIS HYLAE (Johnston 1912) Edmonds 1957}

\section{(=Echinorhynchus hylae Johnston 1912 = Echinorhynchus bulbocaudatus Southwell et Macfie 1925 = Gordiorhynchus hylae (Johnston 1912) Johnston et Edmonds 1948).}

Provenance: Adultes dans l'intestin grêle de Centropus phasianinus (Latham) à Townsville (Queensland) et de Podargus strigoides à Orrorro (Australie du Sud). Juvéniles enkystés dans le mésentère de Hyla aurea Lesson (Nouvelles-Galles-du-Sud), Hyla caerulea White (région de Brisbane) et Limnodynastes dorsalis (fig. 11) (Adelaïde) (cf. Johnston 1912 et 1914 ; J. Burton-Cleland 1922 ; Johnston et Edmonds 1948, Edmonds 1957 et Southwell et Macfie 1925).

\section{Deseription}

«Corps long, cylindrique et plus large dans sa partie postérieure. Dans les deux sexes, pseudo-segmentation interne. Extrémité postérieure des femelles plus dilatée et arrondie. Près de la vulve, présence d'un petit appendice épidermique. Mâles de 48 à $52 \mathrm{~mm}$ de long sur 1,1 à $1,3 \mathrm{~mm}$ de diamètre maximum. Femelles de 56 à $81 \mathrm{~mm}$ de long sur 1,4 à $1,8 \mathrm{~mm}$ de diamètre maximum mesuré immédiatement en avant de la dilatation postérieure. Le diamètre de ce bulbe terminal est de 1,9 à $2,5 \mathrm{~mm}$. Proboscis petit eu égard à la taille du ver, long de 0,55 à $0,65 \mathrm{~mm}$ pour un diamètre maximum de 0,37 à $0,43 \mathrm{~mm}$. Au niveau de l'extrémité antérieure sa largeur est de 0,12 à $0,13 \mathrm{~mm}$. Cou court, inerme, de 0,10 à $0,15 \mathrm{~mm}$ de haut sur $0,30 \mathrm{~mm}$ de diamètre. Rostre armé de 26 à 28 files longitudinales de dix crochets chacune. Les crochets $\mathrm{I}$ à $\mathrm{V}$ sont les plus gros et possèdent des racines bien développées à direction postérieure. Les racines des épines VI à VII sont plus petites et une apophyse à direction antérieure apparaît. Les épines VIII, IX et X n'ont plus que cette apophyse antérieure. Réceptacle à double paroi inséré à la hauteur de la couronne basale d'épines. La paroi du réceptacle a $0,02 \mathrm{~mm}$ d'épaisseur et la longueur du réceptacle est de 1,1 à 1,6 mm. Lemnisques d'environ 3 à $4 \mathrm{~mm}$ de long. Système lacunaire bien développé. Tronc à paroi épaisse dans laquelle on ne trouve aucun noyau. Les coupes transversales montrent, dans les deux sexes, une pseudo-segmentation interne. Testicules longs, ellipsoïdaux, approximativement égaux, placés l'un derrière l'autre dans la moitié antérieure du ver, longs de 1,2 à $2,2 \mathrm{~mm}$ 

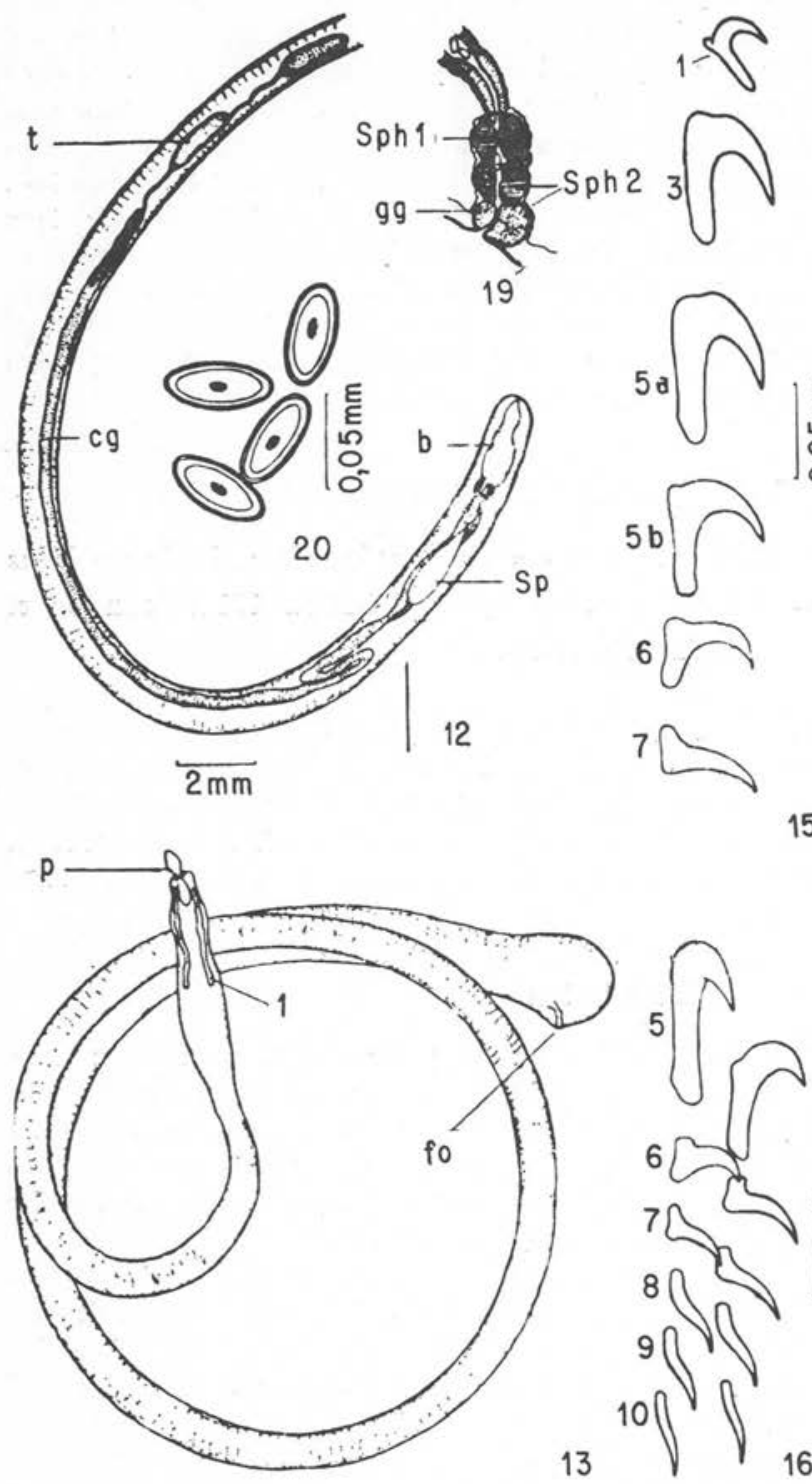

15
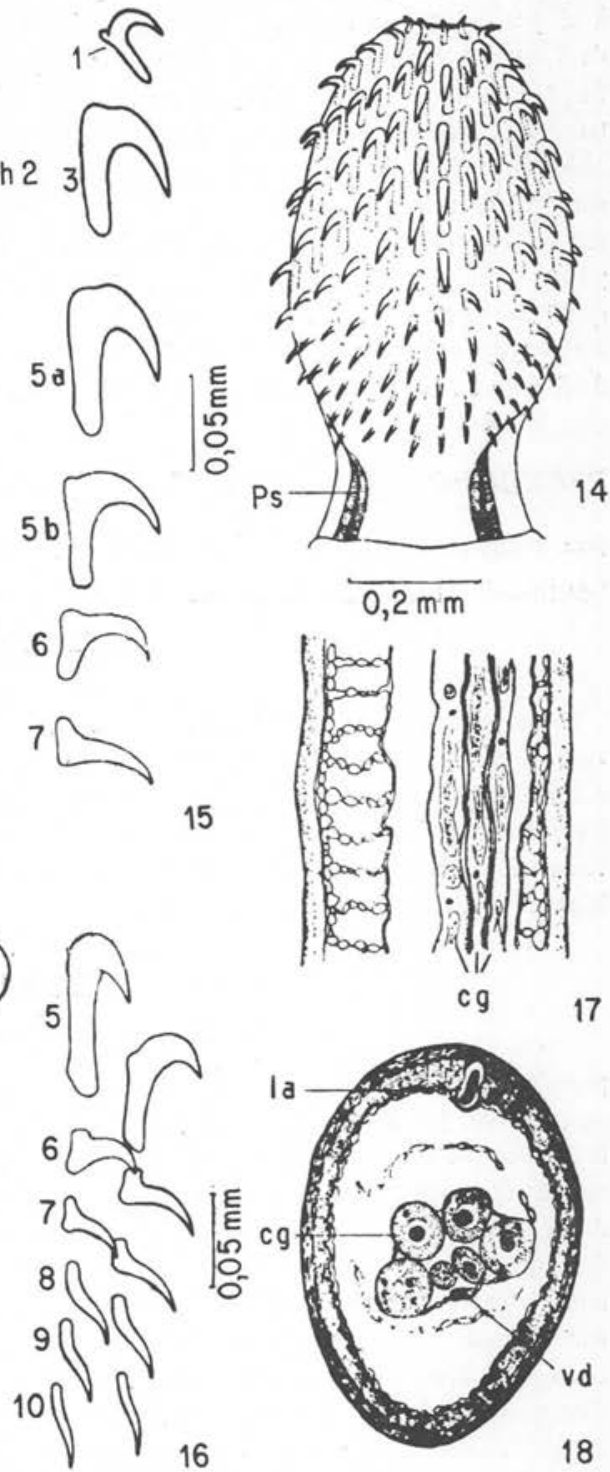

14
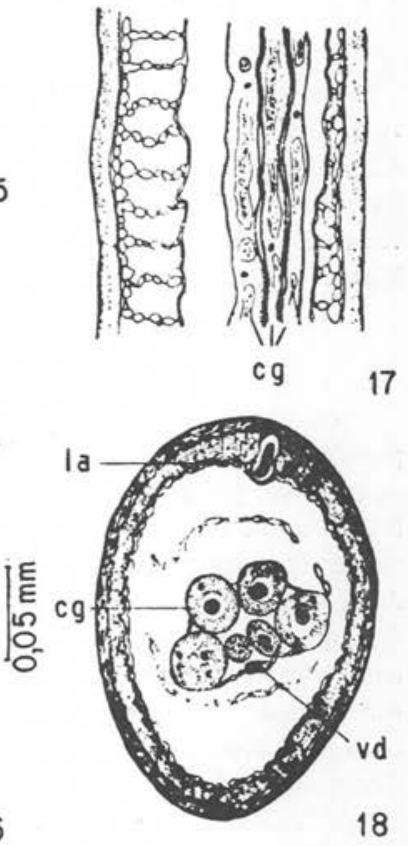

Fig. 11. - Pseudoporrorchis hylae (Johnston 1912) Edmonds 1957. Reproduction des dessins originaux d'Edmonds : 12) partie terminale du corps d'un mâle; 13) femelle adulte; 14) proboscis; 15) crochets du rostre d'un individu femelle; 16) crochets du rostre montrant les changements de forme des racines ; 17) pseudo-segmentation interne d'un individu mâle ; 18) coupe transversale d'un mâle montrant les six glandes cémentaires; 19) partie terminale de l'appareil génital femelle ; 20) embryophore 
sur 0,42 à $0,60 \mathrm{~mm}$ de diamètre maximum. Six glandes cémentaires tubuleuses, en groupe compact, dont quatre naissent près du testicule postérieur. Organe de Säfftigen $(=$ " cement reservoir" dans le texte) long. Bourse portant deux diverticules antérieurs. Orifice génital mâle terminal. Utérus caché par les œufs et les masses ovariennes. Vagin formé de trois bulbes. Vulve subterminale marquée par une dilatation de la cuticule. Embryophores mûrs ellipsoïdaux, ayant de 0,054 à $0,060 \mathrm{~mm}$ de long sur 0,024 à $0,028 \mathrm{~mm}$ de diamètre (montage au Baume du Canada), sans hernies polaires. »

Edmonds (1957) a revu les types de l'Echinorhynchus bulbocaudatus de Southwell et Macfie qui sont identiques à $P$. hylae. En ce qui concerne l'Echinorhynchus centropusi de Tubangui, Edmonds écrit : "Further, through the kindness of the late Professor H.J. Van Cleave, five slides of Pseudoporrorchis centropusi (Tubangui 1933), all named by Tubangui, were made available for re-examination. A study of these specimens showed that the range of measurements of some organs and structures of $P$. centropusi could be extended, e. g. (1) the lengh of the male may be as long as $21 \mathrm{~mm}$ and the female $28 \mathrm{~mm}$, (2), the introvert is armed with 26 longitudinal rows of $8-10$ hooks per row, and (3) ripe eggs about $0,050 \times 0,023 \mathrm{~mm}$ are present in one female. In addition, internal pseudosegmentation is present and the female aperture is sub-terminal. This extra information brings Tubangui's specimens from Centropus viridis into the synonymy of $P$. bulbocaudatus. »

Nous sommes parfaitement en accord avec Edmonds pour considérer que l'Echinorhynchus bulbocaudatus de Southwell et Macfie est un parfait synonyme de l'E. hylae de Johnston. Nous pensons également qu'il a, avec raison, considéré l'E. centropusi de Tubangui comme synonyme de l'E. hylae. Mais comme l'on ne connaît pas avec exactitude le nombre de glandes cémentaires des mâles de l'espèce des Philippines, nous souhaiterions que ce point fût précisé avant que cette mise en synonymie ne devienne définitive.

$$
* * *
$$

\section{PSEUDOPORRORCHIS MAXVACHONI n. sp.}

Provenance: Intestin grêle d'un Courol (Leptosomus discolor $=$ Vorondreo en malgache) $\left(\mathrm{n}^{\circ}\right.$ By. 249-T. 158) tué à Tananarive dans le parc de l'Institut Pasteur le 18 avril 1959.

\section{Description}

Nous disposons de deux exemplaires seulement, un mâle et une femelle, mûrs, et en excellent état de conservation.

《 Mâle de $41 \mathrm{~mm}$ de long sur 2,40 $\mathrm{mm}$ de diamètre dans son sixième antérieur dilaté et $2 \mathrm{~mm}$ de diamètre dans le reste du tronc, femelle de $55 \mathrm{~mm}$ de long pour un diamètre de $2,25 \mathrm{~mm}$ dans la dilatation antérieure, $1,90 \mathrm{~mm}$ dans la partie moyenne et 2,60 $\mathrm{mm}$ dans la partie postérieure dilatée en bulbe (fig. 12-A, B). Rostre sphéroïdal de $1 \mathrm{~mm}$ de long sur $0,80 \mathrm{~mm}$ de diamètre chez la femelle et de $0,85 \mathrm{~mm}$ de long sur $0,70 \mathrm{~mm}$ de diamètre chez 

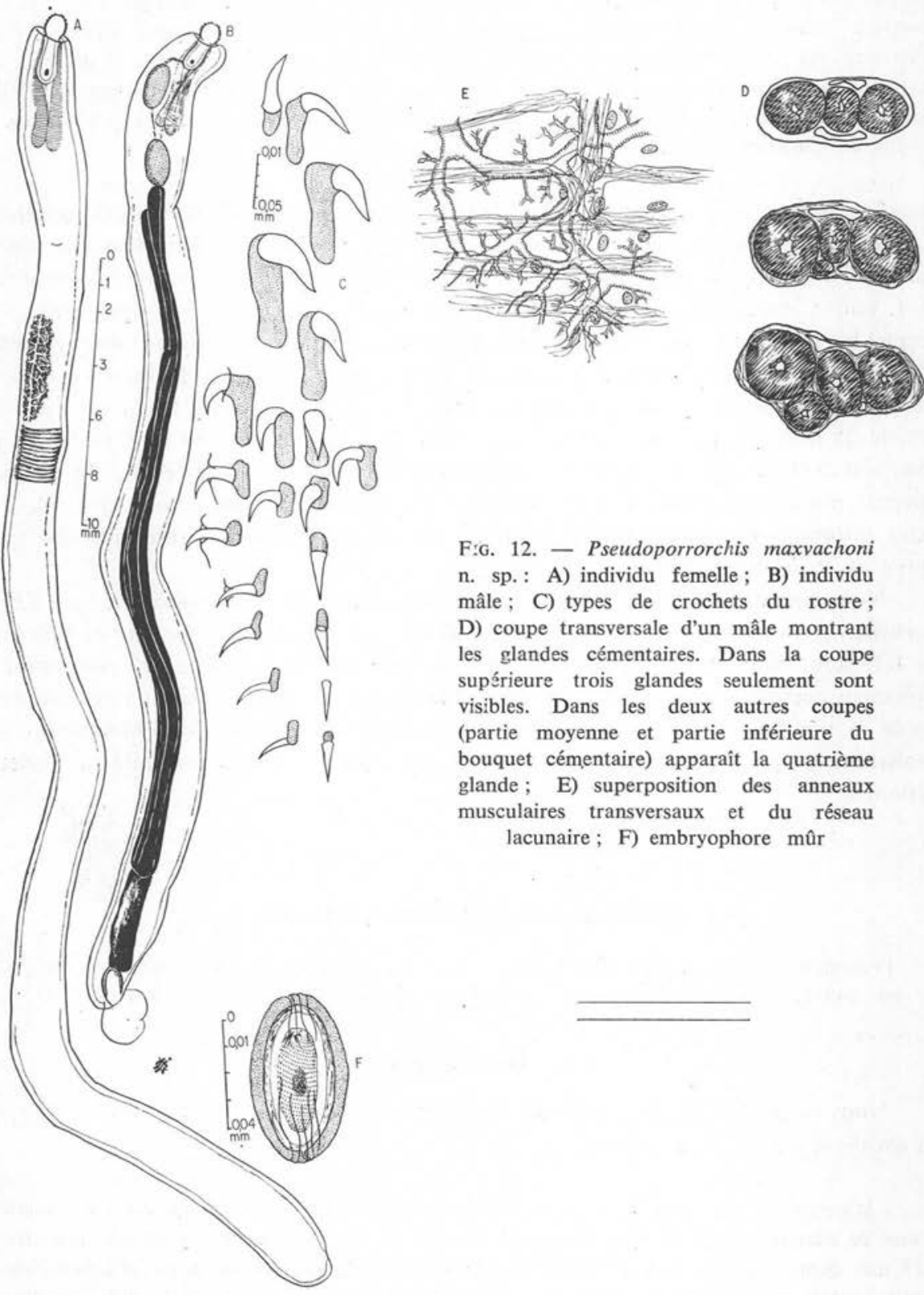

F:G. 12. - Pseudoporrorchis maxvachoni n. sp. : A) individu femelle; B) individu mâle; C) types de crochets du rostre; D) coupe transversale d'un mâle montrant les glandes cémentaires. Dans la coupe supérieure trois glandes seulement sont visibles. Dans les deux autres coupes (partie moyenne et partie inférieure du bouquet cémentaire) apparait la quatrième glande; E) superposition des anneaux musculaires transversaux et du réseau lacunaire; F) embryophore mûr 
le mâle, armé de 40 à 44 files longitudinales de trois à quatre crochets vrais antérieurs et cinq ou six épines postérieures (fig. 13). Les crochets ont tous une racine de forme simple (fig. 12-C), avec une légère dilatation bulboïde postérieure et une lame bien plus fortement arquée vers son extrémité libre que vers sa base. La taille des crochets croît rapidement du I au III qui est le plus gros puis diminue ensuite. Les dimensions sont les suivantes, mesurées sur le rostre du mâle : crochet $\mathrm{I}$ : lame de $0,055 \mathrm{~mm}$ de long sur $0,018 \mathrm{~mm}$ de large à la base, avec une racine de $0,025 \mathrm{~mm}$ de long; II : lame de $0,060 \mathrm{~mm}$ de long sur $0,02 \mathrm{~mm}$ de large et racine de $0,050 \mathrm{~mm}$ de long; III : lame de $0,060 \mathrm{~mm}$ de long sur $0,025 \mathrm{~mm}$ de large et racine de $0,100 \mathrm{~mm}$ de long; III bis: lame de $0,080 \mathrm{~mm}$ de long sur $0,030 \mathrm{~mm}$ de large et

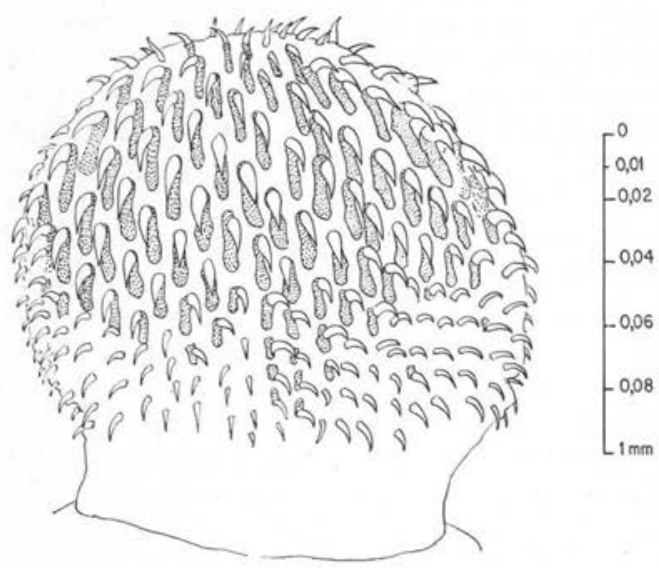

Fig. 13. - Pseudoporrorchis maxvachoni n. sp. rostre

FIG. 14. - Pseudoporrorchis maxvachoni n. sp.: praesoma d'un mâle

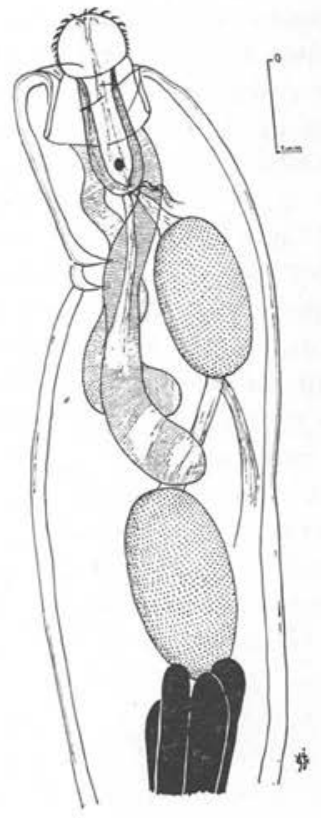

racine de $0,115 \mathrm{~mm}$ de long; IV : lame de $0,055 \mathrm{~mm}$ de long sur $0,025 \mathrm{~mm}$ de large et racine de $0,09 \mathrm{~mm}$ de long; IV bis: lame de $0,050 \mathrm{~mm}$ de long sur $0,020 \mathrm{~mm}$ de large et racine de $0,065 \mathrm{~mm}$ de long.

Les épines sont bien plus minces que les crochets et présentent une courbure régulière depuis leur base d'insertion jusqu'à leur pointe. L'épine $\mathrm{V}$, qui est la plus grosse, possède une racine avec une apophyse supérieure et une inférieure. Les épines VI, VII, VIII et IX n'ont plus qu'une seule apophyse supérieure et pas d'apophyse inférieure sur la racine, l'épine X n'a plus qu'une toute petite base d'insertion granuleuse. Les dimensions des épines sont les suivantes: épine $\mathrm{V}$ : lame de $0,045 \mathrm{~mm}$ de long sur $0,015 \mathrm{~mm}$ de large et racine de $0,03 \mathrm{~mm}$ de long; épine VI : lame de $0,042 \mathrm{~mm}$ de long sur $0,012 \mathrm{~mm}$ de large et racine de $0,02 \mathrm{~mm}$ de long; épine VII : lame de $0,045 \mathrm{~mm}$ de long sur $0,010 \mathrm{~mm}$ de large et racine de $0,023 \mathrm{~mm}$ de long; épine VIII : lame de $0,043 \mathrm{~mm}$ de long sur $0,010 \mathrm{~mm}$ de large et racine de $0,025 \mathrm{~mm}$ de long; épine IX : lame de $0,035 \mathrm{~mm}$ de long sur $0,008 \mathrm{~mm}$ de large 
et racine de $0,023 \mathrm{~mm}$ de long; épine $\mathrm{X}$ : lame de $0,035 \mathrm{~mm}$ de long sur $0,007 \mathrm{~mm}$ de large et racine de $0,015 \mathrm{~mm}$ de long.

Cou tronc-conique de $0,80 \mathrm{~mm}$ de long sur $1,05 \mathrm{~mm}$ de large à sa base. Réceptacle à double paroi inséré au niveau de la dernière couronne d'épines, long de $1,30 \mathrm{~mm}$ en moyenne, pour un diamètre de $0,55 \mathrm{~mm}$. Ganglion cérébroïde placé un peu à distance du fond du réceptacle. Lemnisques longs et aplatis, à grosse extrémité inférieure, dépassant très nettement le fond du réceptacle et atteignant le testicule postérieur (fig. 14). Ils ont environ $3,8 \mathrm{~mm}$ de long chez le mâle et $4 \mathrm{~mm}$ de long chez la femelle. Tronc inerme, présentant une pseudo-segmentation due à la puissance des anneaux musculaires de l'assise pariétale circulaire. Canaux principaux du système lacunaire latéraux, reliés par un réseau d'anastomoses à mailles grossièrement polyédriques (fig. 12-E). Testicules ovoïdes, placés dans la partie antérieure dilatée du tronc, l'un derrière l'autre, et non en contact, mesurant de 1,80 à $2 \mathrm{~mm}$ de long sur environ $1 \mathrm{~mm}$ de diamètre, le postérieur étant un peu plus gros que l'antérieur. Quatre glandes cémentaires très longues, dont deux naissent immédiatement er ^dessous du testicule postérieur, la troisième un peu plus bas et la quatrième nettement plus bas (fig. 12-D). Toutes les quatre se dilatent un peu au-dessus de l'organe de Säfftigen. Ce dernier mesure environ $0,35 \mathrm{~mm}$ de long, il est piriforme, à grosse extrémité supérieure, et flanqué ventro-latéralement par les canaux cémentaires dilatés. Pénis tronc-conique, centrant la bourse copulatrice, laquelle est renforcée par une quinzaine de rayons digitiformes et est appendue en position ventro-subterminale à l'extrémité postérieure du tronc. Appareil génital femelle (fig. 15-A, B) petit par rapport à la longueur du corps, comprenant une cloche utérine de $0,30 \mathrm{~mm}$ de long sur $0,30 \mathrm{~mm}$ de large, centrée par le ligament antéro-postérieur. Portion tubulaire dilatée dans sa partie supérieure, se rétrécissant ensuite, longue de $1,20 \mathrm{~mm}$ de long approximativement. Vagin possédant deux forts sphincters. Vulve ventro-subterminale. Embryophores mûrs mesurant environ $0,060 \mathrm{~mm}$ de long sur $0,030 \mathrm{~mm}$ de diamètre, à coque externe épaisse ornée de saillies vermiculées partant des deux pôles. Pas de hernies polaires de la membrane moyenne. Acanthor de $0,04 \mathrm{~mm}$ de long sur $0,018 \mathrm{~mm}$ de diamètre, orné d'épines sur toute la surface du tégument (fig. 12-F). »

\section{Discussion}

Lorsque l'on compare des spécimens mûrs de Pseudoporrorchis rotundatus et ceux du «Vorondreo » on est immédiatement frappé par la grande différence de taille entre les deux espèces, en particulier par le grand diamètre des seconds. L'examen du rostre vient confirmer la différence puisqu'il n'y a que 28 à 32 files de dix à onze crochets chez l'espèce des Centropus et des Coua alors qu'on compte 40 à 44 files de huit à dix crochets chez le parasite du Leptosomus. Ces deux espèces malgaches sont donc fort différentes l'une de l'autre.

D'ailleurs, l'espèce du «Vorondreo » est, de toutes les espèces actuellement décrites, celle qui possède le plus grand nombre de files de crochets sur le rostre.

Nous considérons donc cette espèce comme nouvelle et nous proposons pour elle le nom de Pseudoporrorchis maxvachoni, en hommage à notre collègue, le Professeur Max Vachon, du Muséum d'Histoire naturelle de Paris. 
$\mathrm{Si}$ nous résumons dans un tableau les caractères morphologiques principaux des dix espèces du genre on voit que les espèces afroasiatiques possèdent toutes quatre glandes cémentaires alors que les espèces australiennes en possèdent toutes six. Il eût été intéressant de connaître le nombre exact de glandes cémentaires de $P$. centropusi et de $P$. te'iger en raison de leur provenance géographique au voisinage de la ligne de Wallace. Il faudra donc revoir ces espèces et faire des coupes transversales de la partie terminale du tronc des mâles pour trancher cette question.

Nous pensons cependant que l'on peut d'ores et déjà proposer la création de deux sous-genres :
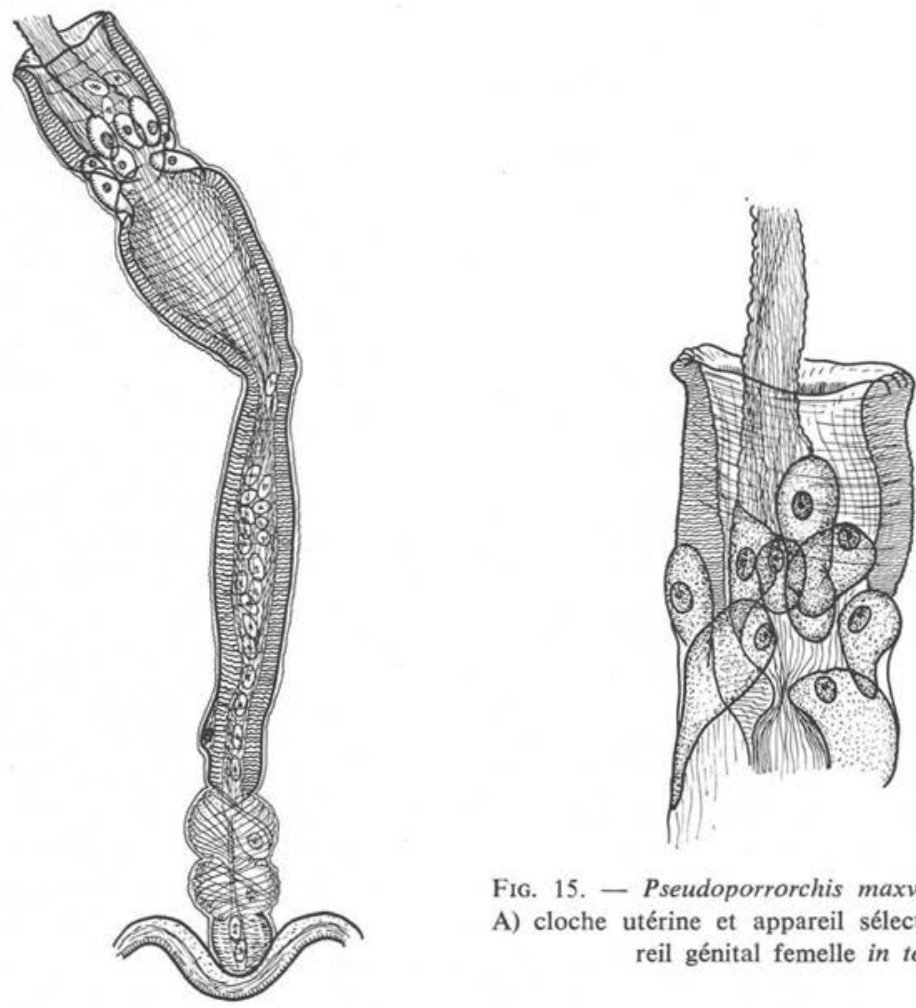

Fig. 15. - Pseudoporrorchis maxvachoni n. sp. :

A) cloche utérine et appareil sélecteur; B) appareil génital femelle in toto

- le premier, Pseudoporrorchis, parasite de Cuculliformes tropicaux afro-asiatiques, possède quatre glandes cémentaires, le type du genre, $P$. houdemeri est aussi le type de ce sous-genre ;

- le second, Austroporrorchis n.s.-gen., parasite de Cuculliformes de la région australienne, possède six glandes cémentaires avec $P$. hylae comme type.

Les deux espèces $P$. teliger et $P$. centropusi demeurent pour le moment inclassables, puisqu'on ne connaît pas le nombre exact des glandes cémentaires de mâles. 


\begin{tabular}{|c|c|c|c|c|}
\hline & $\begin{array}{l}\text { NOMBRE } \\
\text { DE FILES } \\
\text { LONGITU- } \\
\text { DINALES } \\
\text { DE CROCHETS }\end{array}$ & $\begin{array}{c}\text { NOMBRE } \\
\text { DE CROCHETS } \\
\text { PAR FILE }\end{array}$ & $\begin{array}{c}\text { NOMBRES } \\
\text { RESPECTIFS } \\
\text { DE CROCHETS } \\
\text { ET D'ÉPINES }\end{array}$ & $\begin{array}{l}\text { NOMBRE } \\
\text { DE GLANDES } \\
\text { CÉMENTAIRES }\end{array}$ \\
\hline P. houdemeri & 22 à 24 & 11 à 12 & $6+6$ & 4 \\
\hline P. centropi .. & 20 à 24 & 10 à 12 & $6(7)+4(5)$ & 4 \\
\hline$P$. rotundatus. & 28 à 32 & 10 à 11 & $5+5(6)$ & 4 \\
\hline P. teliger .... & 24 à 28 & 9 à 10 & $3+6(7)$ & inconnu \\
\hline$P$. indicus ... & 20 à 24 & 8 à 9 & $4(5)+4(5)$ & 4 \\
\hline P. bazae ... & 38 à 41 & 12 à 13 & inconnus & 6 \\
\hline P. hydromuris & 26 & 7 à 8 & $4+4$ & 6 \\
\hline P. centropusi & $\begin{array}{c}16 \text { à } 18 \\
26 \text { fide Edmonds }\end{array}$ & 8 à 10 & inconnus & inconnu \\
\hline P. hylae $\ldots$. & 26 à 28 & 10 & $5+5$ & 6 \\
\hline P. maxvachoni & 40 à 44 & 8 à 10 & $3(4)+5(6)$ & 4 \\
\hline
\end{tabular}

\section{Résumé}

Dans la présente note nous donnons l'essentiel des descriptions des dix espèces que compte actuellement le genre Pseudoporrorchis Joyeux et Baer 1935. Nous décrivons une nouvelle espèce malgache, parasite de l'intestin grêle du *Vorondreo » (Leptosomus discolor), qui diffère de toutes les autres espèces décrites jusqu'à ce jour par sa grande taille et surtout le grand nombre de files de crochets qui arment son rostre.

La constatation que les espèces afro-asiatiques du genre possèdent quatre glandes cémentaires alors que celle de la région australienne en ont six, nous amène à proposer de scinder le genre en deux sous-genres.

- Pseudoporrorchis afro-asiatiques, à quatre glandes cémentaires ;

- Austroporrorchis n.s.-g. australiens, à six glandes cémentaires.

Les deux espèces $P$. centropusi (Tubangui 1933) des Philippines et $P$. teliger Van Cleave 1949 de Java, dans lesquelles ce nombre de glandes n'est pas connu, doivent, provisoirement, ne pas prendre place dans l'un de ces sous-genres. 


\section{Bibliographie}

Burton-Clealand (J.), 1922. - The parasites of australian Birds. Transac. Roy. Soc. South Australia, XLVI, 85, 118.

DAS (E.-N.), 1957. - On juvenile and adult forms of Pseudoporrorchis indicus, a new species of Acanthocephala from India. Jl. Parasitolog. XLIII (6), 659-663.

Edmonds (S.-J.), 1957. - Australian Acanthocephala. Part X. Transac. Roy. Soc. South Australia, LXXX, 76-80, fig. 1-5.

FUKUI (T.), 1929. - On some Acanthocephala found in Japan. Annotat. Zoolog. Japonens., XII (1), 225-270.

GolvaN (Y.-J.), 1956. - a) Acanthocéphales d'Oiseaux. Quatrième note. Considérations sur le genre Pseudoporrorchis Ch. Joyeux et J.-G. Baer 1935 (Polymorphidae-Porrorchinae) et redescription de Pseudoporrorchis centropi (A. Porta 1910). Bull. Soc. Zoolog. France, LXXXI (1), 62, 71 (+ fig. 1-8). - b) Acanthocéphales d'Oiseaux. Note additionnelle. Pseudoporrorchis rotundatus (O. v. Linstow 1897) (Palaeacanthocephala-Polymorphidae) parasite d'un Cucullidae, Centropus madagascariensis (Briss.) Ibid., LXXXI (5/6), 339-344 (+ 1 fig. et 1 pl.).

-, 1960. — Le Phylum des Acanhocephala. Troisième note. La Classe des Palaeacanthocephala (Meyer 1931). Ann. Parasitol. Hum. Compar., XXXV (1/2), 138-165 (+ fig ; $1-18)$.

—, 1960. - Id. (suite). Ibid., XXXV (3), 350-386 (fig. 19-38).

-, 1960. — Id. (suite). Ibid., XXXV (4), 573-593 (fig. 39-47).

-, 1960. — Id. (suite). Ibid., XXXV (5/6), 713-723 (fig. A, B et C).

—, 1961. — Id. (suite). Ibid., XXXVI (1/2), 76-91.

—, 1961. — Id. (suite). Ibid., XXXVI (4), 612-647.

$\longrightarrow$ 1961. — Id. (suite). Ibid., XXXVI (5/6), 717-736.

Johnston (T. H.), 1912. - Notes on some Entozoa. Proceed. Roy. Soc. Queensland, XXIV, 63-91.

—, 1914. - Some new Queensland Endoparasites. Proceed. Roy. Soc. Queensland, XXVI, 76-84.

Johnston (T. H.) et Edmonds (S. J.), 1948. - Australian Ancanthocephala. Part. VII. Transac. Roy. Soc. South Australia, LXXII (1), 69-76 (fig. 1-20).

Joyeux (Ch.) et BAer (J.-G.), 1935. - Etude de quelques Acanthocéphales d'Indochine. Ann. Mus. Hist. Nat. Marseille, XXVII (mém. $\mathrm{n}^{\circ} 2$ ), 1-15 (+ fig. 1-8).

LiNSTow (O. voN), 1897. - Nemathelminthen, grösstenteils in Madagascar gessammelt. Archiv. f. Naturg. Jahrg., I (1), 27-34 (+ Taf. IV u. V).

Marval (L. DE), 1905. - Monographie des Acanthocéphales d'Oiseaux. Thèse Sci. Lausanne, publ. in Rev. Suisse Zoolog. Genève, XII, 195-387 (+ 4 pl.).

MeYer (A.), 1931. - Neue Acanthocephalen aus dem Berliner Museum. Begründung eines neuen Acanthocephalensystems auf Grund einer Untersuchungen der Berliner Sammlung. Zoolog. Jahrb. System. Geograph., LXII (1/2), 53, 108 (+ 74 fig.).

Petrotschenko (V.-I.), 1956. - Acanthocéphales des animaux sauvages et domestiques, Tome I, 435 pp. (+ fig. 1-181) (en russe). 
-, 1958. - Tome II, 458 pp. (+ fig. 1-178) (en russe).

Porta (A.), 1910. - Acantocefali nuovi o poco noti. Zoolog. Anzeig. XXXV (22), (24), 699 703 (+ fig. 1-4).

Southwell (T.) et MAcFie (J.-W.-S.), 1925. - On a collection of Acanthocephala in the Liverpool School of Tropical Medicine. Ann. Trop. Med. Parasitol., XIX (2), 141184.

Tubangur (M.-A.), 1933. - Notes on the Acanthocephala in Philippines Philippines Jl. Sci., L (2), 115-128 (+ 6 pl.).

Van Cleave (H. J.), 1949. - Pseudoporrorchis teliger, a new species of Acanthocephala from Java. Parasitol., XLIX (3/4), 214-217 (+ 1 pl.).

YAMAguti (S.), 1935. - Studies on the helminth fauna of Japan. Part VIII Acanthocephala 1. Japanese. Jl. Zoolog., VI (2), 247-278 (+ fig. 1-30).

-, 1963. - Systema Helminthum. Tome V: Acanthocephala.

Institut de Parasitologie de la Faculté de Médecine de Paris

(Direct. : Prof. Lucien Ch. BRUMPT)

et Institut Pasteur de Madagascar (Direct. : Edouard.-R. BRYGoo). 